\title{
Effects in polarimetry of interference within wave plates
}

\author{
D. Clarke
}

\author{
Department of Physics and Astronomy, The University, Glasgow G12 8QQ, Scotland, UK \\ e-mail: d.clarke@astro.gla.ac.uk
}

Received 27 October 2004 / Accepted 10 January 2005

\begin{abstract}
Multiple-beam interference within wave plates is investigated in terms of the detrimental effects it produces in the data of stellar spectropolarimetry. It is noted that spectral fringe structures occur in the phase delay, the polarizance and, for Pancharatnam designs, the reference axis of the wave plate. The natures of the problems are exposed by considering typical wave plates and experimental procedures used in linear and circular spectropolarimetry. It is demonstrated that the chief bane of accurate measurements is the presence of polarizance fringes, but which can be alleviated by the choice of experimental procedure. For spectral circular polarization studies, problems of cross-talk from any linear polarization present in the source are especially severe. In principle the effects of fringing can be removed in data reductions by calibration measurements of a set of linear polarization standard stars displaying different vibration azimuths and, for circular polarization measurements, knowledge of the linear polarization characteristics of the investigated star must also be known.
\end{abstract}

Key words. instrumentation: polarimeters - techniques: polarimetric

\section{Introduction}

One of the common ways of performing stellar polarimetry is by the application of a modulator comprising a rotatable wave plate prior to a fixed polarizer. For measurements of linear polarization, the plate invariably has a half-wave retardation, while, for the measurement of circular polarization, the plate is normally of quarter-wave design. One of the advantages of this kind of system is that the polarizer remains fixed in the instrument, together with the detector. With such an arrangement, spurious signals are not generated by any sensitivity of the detector to the azimuth of the polarization falling on it, as this is constant. By employing two materials such as quartz and magnesium fluoride, achromatic wave plates are commonly used and, in the case of half-wave devices, a Pancharatnam (1955) configuration is often employed to provide a superachromatic modulator (see the recipe of Serkowski 1974). For the previous generation of polarimeters using photomultipliers as detectors, measurements were made either by continuously rotating the wave plate or by integrating the transmitted intensity values at preferred fixed orientational settings corresponding to the techniques of Pickering (1874) or Fessenkov (1935) (see Clarke \& Grainger 1971). A recommended technique for measurement of the signal produced by a modulator involving a continuously rotating wave plate is described by Klare et al. (1972) and it has been used in instruments constructed at Glasgow University Observatory (e.g., see Clarke et al. 1985; Clarke \& Fullerton 1996). As for the polarizer, this may be either a single channel device or both resolved components may be transmitted for simultaneous measurement using two detectors. A general description of the principles of stellar polarimetry and the optical elements used has been presented by Serkowski (1974).

As the photomultiplier is essentially a single pixel device, spectral studies could only be undertaken by the insertion of colour filters usually placed in the optical train following the modulator so as not introduce spurious instrumental polarization. In some instruments spectral measurements were possible by sequential wavelength scanning of a monochromator such as a tunable interference filter, e.g., see Clarke et al. (1985). By using achromatic wave plates, the same modulator could be used to undertake spectropolarimetry without need of disturbance as the wavelength pass-band changed by filter selection or by adjustment of the monochromator.

With the advent of two dimensional detectors such as CCDs, the established polarimetric techniques may be adapted. For example, Clarke \& Neumayer (2002) have employed the principle of Klare et al. (1972) to explore the potential of CCDs to improve the signal-to-noise ratios of broad-band polarimetric measurements. Very importantly $2 \mathrm{D}$ detectors allow polarimetry with simultaneous measurements in many spectral channels by using dispersive spectrometers following the modulator system. With such instruments, the spectral resolution can be increased sufficiently to undertake polarimetric studies across stellar line profiles. It has become apparent, however, that "fringes" tend to appear in the data - see, for example, Tinbergen (1994), Harries \& Howarth (1996) and Donati et al. (1999). From their analysis, Harries and Howarth concluded that the observed fringes were consistent with being of the Fabry-Pérot-type producing a fringe-structured instrumental polarization which could be subtracted out by reference 
observations of unpolarized standard stars. Problems generated by spurious signals of this kind would tend to average out in spectral measurements involving broad-band filters and appear not to have been encountered in polarimetry using single pixel photoelectric detectors. Even at higher spectral resolution, as achieved in the tilt-scanning narrow-band interference filter systems as used by Clarke et al. (1985), effects of fringes appear to have been bypassed as a result of the data recording technique (see later discussion).

In a recent paper, Semel (2003) has reviewed the circumstances whereby polarized radiation can be subject to interference. Part of the discussion was related to multiple-beam interference in wave plates and a first-order theory was presented to describe the behaviour of the engendered fringes. In the spectropolarimetric instrumental development of Donati et al. (1999), it was found that the strength of the fringes depended on the choice of plate manufacturer. They also noted that the analytic modelling as proposed by Harries \& Howarth (1996) for their data was successful at a spectral resolution, $R$ of $5 \times$ $10^{3}$, but would be insufficient at $R=35 \times 10^{3}$ as a result of the very complex shapes of the multiperiodic behaviour occurring in the fringes.

In this paper, the nature of the fringes caused by interference within wave plates is explored more comprehensively to see how they generate spurious signals in basic spectropolarimetry associated with the measurement of linear and circular polarized light and how their effects might be minimized by experimental procedures.

\section{The nature of wave plates}

Wave plates alter the polarization state of light transmitted through them by introducing a relative phase delay between the principal axes referred to as "fast" and "slow", these being orthogonal to each other. Generally wave plates are manufactured from uniaxial birefringent crystals cut so that the ordinary, $n_{\mathrm{o}}$, and extraordinary, $n_{\mathrm{e}}$, refractive indices relate to the principal axes. The ascribed phase delay is defined as the difference in optical path length for the transmitted electric components resolved parallel to these axes. According to standard optical texts, the phase delays, $\phi_{\mathrm{s}}, \phi_{\mathrm{f}}$, associated with the slow and fast principal axes, may be written as

$\phi_{\mathrm{s}}=\frac{2 \pi d n_{\mathrm{s}}}{\lambda} \quad$ and $\quad \phi_{\mathrm{f}}=\frac{2 \pi d n_{\mathrm{f}}}{\lambda}$

where $d$ is the thickness of the plate and $n_{\mathrm{s}}, n_{\mathrm{f}}$ the refractive indices for the slow and fast axes, being replaced either by $n_{\mathrm{o}}, n_{\mathrm{e}}$, or by $n_{\mathrm{e}}, n_{\mathrm{o}}$, depending on whether the birefringence is respectively negative or positive. For the case of $n_{\mathrm{e}}>n_{\mathrm{o}}$ (positive birefringence) the nominal relative phase delay is given by

$\phi_{\mathrm{s}}-\phi_{\mathrm{f}}=\delta=\frac{2 \pi d}{\lambda}\left(n_{\mathrm{e}}-n_{\mathrm{o}}\right)$.

Thus, simple theory suggests that a required phase delay, $\delta$, may achieved by cutting and polishing the plate to the required thickness according to the operating wavelength and the values of the refractive indices, $n_{\mathrm{e}}, n_{\mathrm{o}}$, at that wavelength. In most polarimetric instruments, any included wave plate is likely to have a retardation of either $\pi / 2 \equiv \lambda / 4$, or $\pi \equiv \lambda / 2$, based on Eq. (2), although other phase values are sometimes encountered.

In the first of a series of papers on the effects of multiplebeam interference within the wave plate cavity, Clarke (2004a) has applied the Airy formula to demonstrate that a simple retarder provides a phase delay with a value different to the simple expression of Eq. (2).

When multiple reflections within the wave plate are taken into account, the corrected values for the phase delays, $\Phi_{\mathrm{s}}, \Phi_{\mathrm{f}}$, may be determined from the identities

$\tan \Phi_{\mathrm{s}}=\frac{n_{\mathrm{s}}^{2}+1}{2 n_{\mathrm{s}}} \tan \phi_{\mathrm{s}} ; \quad \tan \Phi_{\mathrm{f}}=\frac{n_{\mathrm{f}}^{2}+1}{2 n_{\mathrm{f}}} \tan \phi_{\mathrm{f}}$.

The true phase delay of the wave plate with multiple reflections taken into account may now be written as

$\Delta=\Phi_{\mathrm{s}}-\Phi_{\mathrm{f}}$.

A further issue discussed by Clarke (2004a) is the fact that, in addition to its chief function of introducing a relative phase delay, a wave plate also acts as a weak partial polarizer. Reflection losses at the entrance and exit faces depend on the refractive indices of the plate. As the plate is made of birefringent material, the reflection losses, and hence the transmittances, $\mathcal{T}_{\mathrm{f}}, \mathcal{T}_{\mathrm{s}}$, for the fast and slow orthogonal axes defining the phase delay, have different values. Again, as a result of multiple-beam interference, the values of $\mathcal{T}_{\mathrm{f}}, \mathcal{T}_{\mathrm{s}}$ each display wavelength dependent fringes but with differing spacing according to their associated refractive indices. The intensity transmittance and polarizance of a simple wave plate is therefore likely to display fringes with beat structures resulting from the differences in the fringe spacing associated with the orthogonal axes.

Accordingly, if $\Delta$ is the phase delay and $\mathcal{T}_{\mathrm{f}}, \mathcal{T}_{\mathrm{s}}$ are the intensity transmittances associated with the principal axes, the description of a wave plate in terms of its Mueller matrix may be written as

$$
\left[\begin{array}{cccc}
\mathcal{T}_{\mathrm{f}}+\mathcal{T}_{\mathrm{s}} & \mathcal{T}_{\mathrm{f}}-\mathcal{T}_{\mathrm{s}} & 0 & 0 \\
\mathcal{T}_{\mathrm{f}}-\mathcal{T}_{\mathrm{s}} & \mathcal{T}_{\mathrm{f}}+\mathcal{T}_{\mathrm{s}} & 0 & 0 \\
0 & 0 & 2 \sqrt{\mathcal{T}_{\mathrm{f}} \mathcal{T}_{\mathrm{s}}} \cos \Delta & 2 \sqrt{\mathcal{T}_{\mathrm{f}} \mathcal{T}_{\mathrm{s}}} \sin \Delta \\
0 & 0 & -2 \sqrt{\mathcal{T}_{\mathrm{f}} \mathcal{T}_{\mathrm{s}}} \sin \Delta & 2 \sqrt{\mathcal{T}_{\mathrm{f}} \mathcal{T}_{\mathrm{s}}} \cos \Delta
\end{array}\right]
$$

or

$\left[\begin{array}{cccc}k_{1} & k_{2} & 0 & 0 \\ k_{2} & k_{1} & 0 & 0 \\ 0 & 0 & k_{3} \cos \Delta & k_{3} \sin \Delta \\ 0 & 0 & -k_{3} \sin \Delta & k_{3} \cos \Delta\end{array}\right]$

where $k_{1}=\mathcal{T}_{\mathrm{f}}+\mathcal{T}_{\mathrm{s}}, k_{2}=\mathcal{T}_{\mathrm{f}}-\mathcal{T}_{\mathrm{s}}$ and $k_{3}=2 \sqrt{\mathcal{T}_{\mathrm{f}} \mathcal{T}_{\mathrm{s}}}$. Because of multiple-beam interference, the parameters $k_{1}, k_{2}, k_{3}$ and $\Delta$ all display wavelength dependent fringes.

In a further paper, Clarke (2004b) has developed the algebra associated with the description of multilayer thin-film optical systems and has investigated the problem of interference in compound and achromatic wave plates. As might be expected, the fringe structures associated with the intensity transmittance, polarizance and phase delay become more complex for these devices. The frequency of the fringes with wavelength is also higher resulting from the increased optical thickness relative to single plates, so increasing the order of interference. 


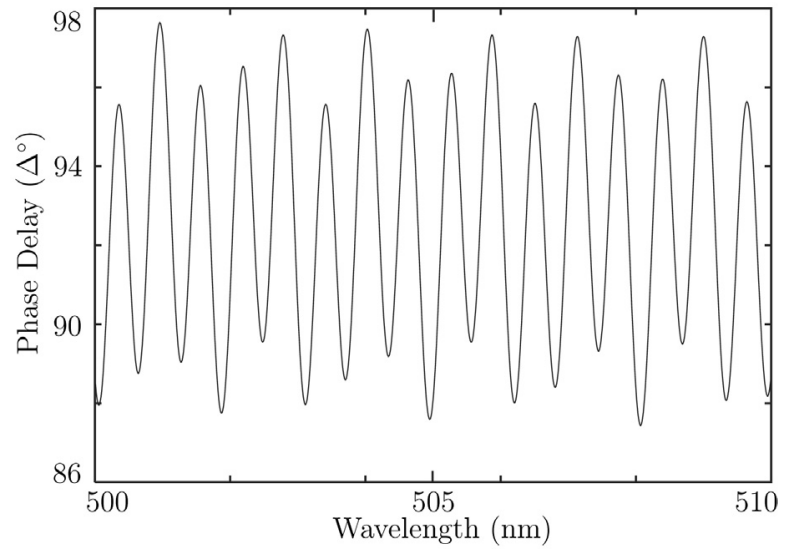

Fig. 1. The phase delay variation of an achromatic quarter-wave plate over a spectral interval of $10 \mathrm{~nm}$. Note that the mean level is greater than $90^{\circ}$, the plate designed to be exactly quarter-wave at $425 \mathrm{~nm}$ and $575 \mathrm{~nm}$. It may be noted that the amplitude of the fringes is greater than the secular departure of the mean from being exactly $90^{\circ}$.

It may be noted that the amplitude of the fringes associated with phase delay may be larger than the displacements of the secular departures for wavelengths away from the two values for which achromaticity is designed. For any compound plate, a single resultant matrix ensues in the form of Eqs. (5) or (6), so describing its behaviour as a retarder in combination with a weak partial polarizer. The exercise also showed that the amplitudes of the various fringes are reduced by the application of anti-reflection coatings to the entrance and exit faces. An assumption was also made that the elements comprising the compound plates were in optical contact. Again, if they were to be bonded, the fringe patterns would be modified and reduced in amplitude.

Finally, in the third paper, Clarke (2004c) further extended the algebra associated with the description of multilayer thinfilm optics to allow calculation of compound wave plates whose elements are set with their principal axes at other than $0^{\circ}$ or $90^{\circ}$ to each other, as in Pancharatnam (1955) designs. In addition to the complex fringe patterns associated with transmission, polarizance and phase delay, it was demonstrated that the angle describing the effective principle axes of such compound plates is also subject to wavelength dependent fringe structures.

For the purpose of exploring the effects of interference associated with a rotating wave plate modulator used to undertake measurements of linear and circular polarization, an achromatic combination of quartz and magnesium fluoride is taken as a "test" device. Following the recipe by Clarke (1967), the thicknesses of the elements were selected to provide the required phase delay at $425 \mathrm{~nm}$ and $575 \mathrm{~nm}$, without consideration of interference effects. For an achromatic half-wave plate, the thicknesses are $157.03 \mu \mathrm{m}$ for quartz and $116.28 \mu \mathrm{m}$ for $\mathrm{MgF}_{2}$; for a quarter-wave plate, the thicknesses of the elements are simply one half of these quoted values. Based on the matrix method of Clarke (2004c) and using values for the refractive indices and their dispersions from Tropf et al. (1995), the characteristics for the half-wave and quarter-wave versions of the achromatic wave plate were calculated, this assuming that the elements were in optical contact with the entrance and exit

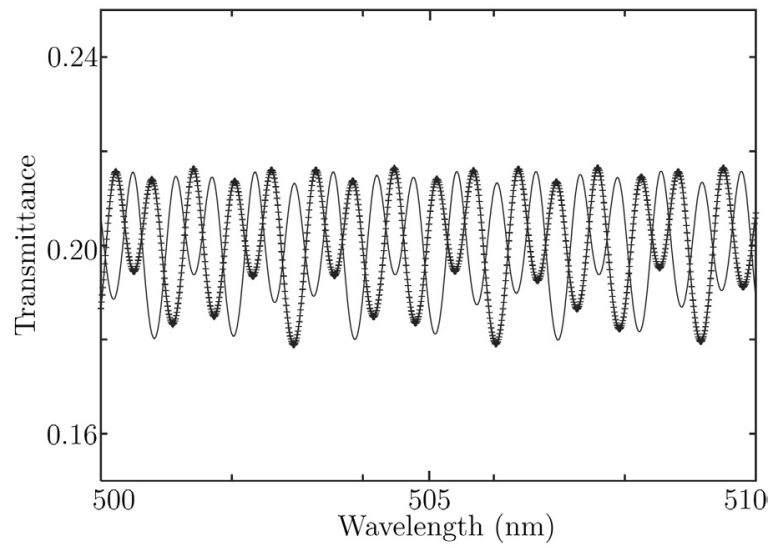

Fig. 2. The variation of the transmittances of the fast axis, $\mathcal{T}_{\mathrm{f}}$, (heavy curve with marked points) and the slow axis, $\mathcal{T}_{\mathrm{s}}$ (light curve) of the same achromatic plate as in Fig. 1. Over this spectral interval of $10 \mathrm{~nm}$, the fringes for the orthogonal axes are nearly in anti-phase, giving rise to polarizance fringes of large amplitude.

surfaces free from anti-reflection coatings. Simply to act as examples, Figs. 1 and 2 illustrate the fringes associated with an achromatic quarter-wave plate over the spectral interval from $500 \mathrm{~nm}$ to $510 \mathrm{~nm}$. In Fig. 1, the phase delay oscillates with a beat pattern of amplitude $\sim 5^{\circ}$, the mean level being some $\sim 2^{\circ}$ above $90^{\circ}$, the displayed wavelength interval lying between the achromatic values. In Fig. 2, the values of $\mathcal{T}_{\mathrm{f}}$ and $\mathcal{T}_{\mathrm{s}}$ each show beating fringe patterns which happen to be close to being in anti-phase with each other. As a consequence, over this spectral interval, the intensity transmittance fringe pattern based on the combination of $\left(\mathcal{T}_{\mathrm{f}}+\mathcal{T}_{\mathrm{s}}\right)$, would be of small amplitude, but those related to polarizance would be most marked.

As noted above, the spectral interval covered in Figs. 1 and 2 lies between the two, key wavelength points selected for the required, exact value of phase delay. This display choice is arbitrary and not aimed to highlight maximum amplitudes for the various fringes. Even around the key wavelength points, the fringe amplitudes are also large. The fringe amplitudes of $\Delta$, of transmittance and of polarizance over a more comprehensive wavelength range all vary but without minima necessarily close to the key designated wavelength points (cf. Figs. 4-6 in Clarke 2004b). Because of multiple beam interference, even at the exact key points, $\Delta$ will be displaced from the design value, according to the position in wavelength of the phase-delay fringe pattern, this in turn dependent on the refractive indices of the selected materials and on the design thicknesses of the individual plates.

In the next section, the effects that the fringe structures have on spectropolarimetric measurements are investigated.

\section{Wave plate polarimetric modulators}

The basic design of any polarimeter involving a rotatable wave plate prior to a fixed polarizer may be expressed in terms of a series of matrices describing the optical elements and their geometric relationships. Suppose that the light for analysis is represented by a Stokes column vector $\{I, Q, U, V\}$ described in the co-ordinate frame of the principal axes of the polarizer. 
The normalised Stokes parameters may be written as $q=Q / I$, $u=U / I$ and $v=V / I$. The linear polarization characteristics of degree of polarization, $p$, and azimuth of vibration, $\theta$, are given by

$p=\sqrt{\left(q^{2}+u^{2}\right)} \quad$ and $\quad \theta=\frac{1}{2} \arctan \frac{u}{q}$.

The degree of circular polarization is simply the value of $v$, with its sign indicating the handedness.

The effects of the optical elements comprising the modulator may by summarised by

$$
\left[\begin{array}{c}
\mathcal{I} \\
Q \\
\mathcal{U} \\
\mathcal{V}
\end{array}\right]=[\text { Pol }]\left[\begin{array}{c}
\text { Rot } \\
(-\alpha)
\end{array}\right]\left[\begin{array}{l}
\text { Wave } \\
\text { Plate }
\end{array}\right]\left[\begin{array}{c}
\operatorname{Rot} \\
(\alpha)
\end{array}\right]\left[\begin{array}{c}
I \\
Q \\
U \\
V
\end{array}\right],
$$

where $\{\mathcal{I}, Q, \mathcal{U}, \mathcal{V}\}$ is the Stokes column vector describing the emergent light falling on the detector. The angle, $\alpha$, is the rotational setting of the axis of the wave plate relative to the principal axis of the polarizer, this remaining fixed in the instrument and used as the reference axis for describing the Stokes vector $\{I, Q, U, V\}$ of the incoming light. It is the intensity $\mathcal{I}(\alpha)$ that is measured as the polarimetric signal.

In Eq. (8) describing the instrument, $[\operatorname{Rot}(\alpha)]$ is a matrix representing rotation of the Stokes vector to the co-ordinate frame describing the wave plate and is given by

$$
\left[\begin{array}{cccc}
1 & 0 & 0 & 0 \\
0 & \cos 2 \alpha & \sin 2 \alpha & 0 \\
0 & -\sin 2 \alpha & \cos 2 \alpha & 0 \\
0 & 0 & 0 & 1
\end{array}\right]
$$

$[\operatorname{Rot}(-\alpha)]$ is a similar matrix involving an angle of opposite sign, returning the Stokes vector to the original co-ordinate frame of the polarizer.

[Pol] is the matrix describing the operation of the fixed polarizer and given by

$$
\frac{1}{2}\left[\begin{array}{llll}
1 & 1 & 0 & 0 \\
1 & 1 & 0 & 0 \\
0 & 0 & 0 & 0 \\
0 & 0 & 0 & 0
\end{array}\right]
$$

The matrix written as [Wave Plate] describes the effect of the wave plate which, for a pure device, simply includes terms describing the effects of the phase delay. In reality, as discussed above, it needs to include the effects of a polarizance. Its form is therefore represented by either Eq. (5) or (6). Using the formulation from the latter equation, and following the multiplying out of the matrices in Eq. (8), the signal for measurement may be organised to be written as

$$
\begin{aligned}
2 \mathcal{I}(\alpha)= & k_{1}(I+Q \cos 4 \alpha+U \sin 4 \alpha) \\
& -k_{3} V \sin \Delta \sin 2 \alpha \\
& +k_{2}(I \cos 2 \alpha+Q \cos 2 \alpha+U \sin 2 \alpha) \\
& +\left(k_{1}+k_{3} \cos \Delta\right)\left(Q \sin ^{2} 2 \alpha-\frac{U}{2} \sin 4 \alpha\right) .
\end{aligned}
$$

In exploring the behaviour of Eq. (9), it would be expected that $k_{1}$ and $k_{3}$ are close to unity with $k_{2}$ close to zero, but all these parameters would exhibit fringing. In the following section, the behaviours of the effects of the various parameters contributing to the signal as expressed in Eq. (9) will be explored by considering the basic measurements of linear and circular polarization in turn. It will be demonstrated that some of the spurious signals generated by the parameters describing a real wave plate can be removed by the chosen method of signal retrieval.

\section{Measurements of linear polarization}

As a check on the behaviour of the signal described in Eq. (9), consider a perfect wave plate with no multiple reflections and with the polarizance neglected. For simplicity it will be assumed that no circular polarization is present and that line 2 of the equation can be ignored. When linear polarization is investigated, the phase delay should be half a wavelength. Hence, $k_{1}=k_{3}=1, k_{2}=0$ and $\Delta=\pi$. With these values the form of the signal reduces to

$\mathcal{I}(\alpha)=\frac{1}{2}\{I+Q \cos 4 \alpha+U \sin 4 \alpha\}$

this coming from the first line of Eq. (9) and is the familiar form expected for such a modulator. When the term $k_{1}$ is included, this chief part of the polarimetric signal is subject to fringing. By itself this has no real consequence as the measurement of the normalised Stokes parameters involves the determination of the ratios $Q / I$ and $U / I$ which are independent of $k_{1}$.

The third line of Eq. (9) comprises spurious contributions given by $I k_{2} \cos 2 \alpha$ and $k_{2}(Q \cos 2 \alpha+U \sin 2 \alpha)$, introduced by the polarizance of the wave plate, the modulation being twice that of its mechanical rotation rate and half that of the chief polarimetric signal. If the source happens to be unpolarized, the strength of this contaminating signal is proportional to the intensity and behaves as though an "instrumental polarization" has been introduced, this being subject to fringing. More generally this instrumental polarization is not a fixed value at any particular wavelength, as would normally be considered in regular polarimetric reduction procedures, but depends on the Stokes parameters of the incoming light being measured. The first element contributing to this spurious signal is, however, likely to be $\sim 100 \times$ stronger than the second, as both $Q / I$ and $U / I$ are generally small, say $\sim 0.01$. Effects of these latter contributions need to be taken into account, however, in high accuracy polarimetry.

It may be noted that the fourth line of Eq. (9) will generally not be zero but close to it, the values of $k_{1}$ and $k_{3}$ both being close to unity, with $\cos \Delta$ being close to -1 . The effect of these terms and their associated fringing therefore affects the modulated amplitudes generated for $Q$ and $U$ as expressed in line 1 of the same equation. The presence of the terms in line 4 describes the defects of modulation efficiency affecting the amplitudes of the signals associated with the $Q$ and $U$ parameters.

When spectropolarimetry is undertaken, fringes affect the wavelength dependent values of $k_{1}, k_{2}, k_{3}$ and $\Delta$ and hence the behaviour of the instrument at each measured wavelength value. The likely effects on the reduced data can be explored by considering one of the standard techniques used for sampling 

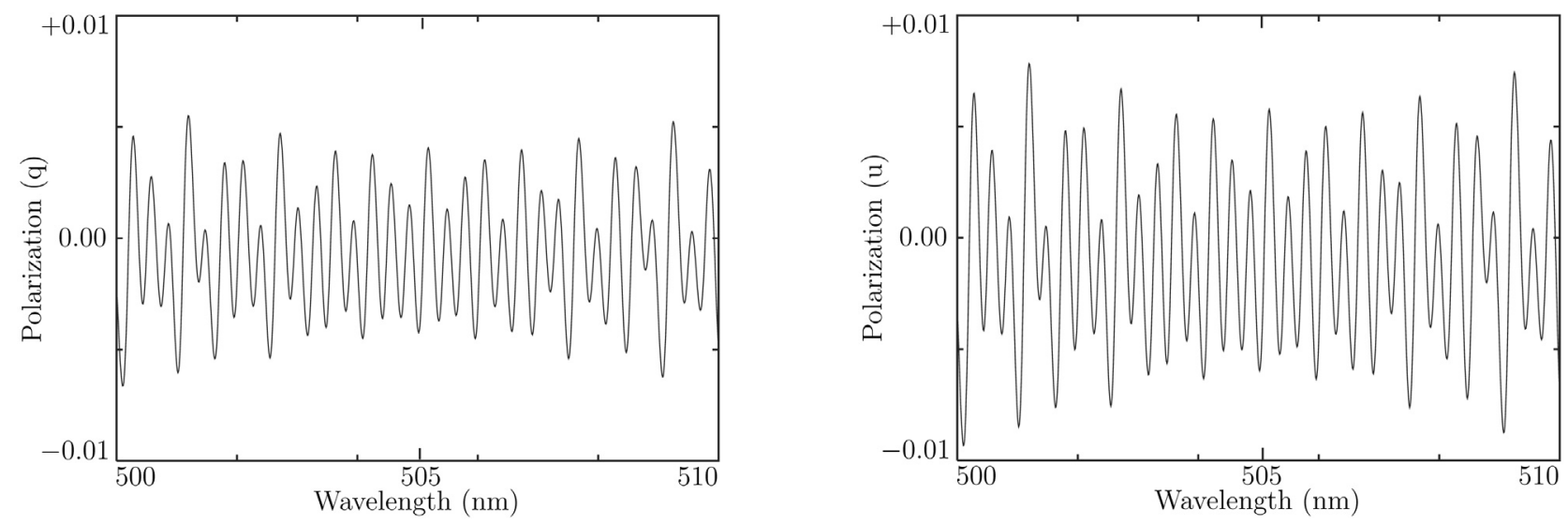

Fig. 3. The variation of the determined values of $q$ and $u$ over a spectral interval of $10 \mathrm{~nm}$ using an achromatic half-wave plate when the analysed light is unpolarized. The fringe structures correspond to the behaviours of Eqs. (15) and (17).

the signal in relation to the characteristics of the achromatic test wave plate as described in Sect. 2 .

A favoured observational procedure corresponds to the Pickering (1874) technique whereby exposures are taken at four angular settings of the wave plate. In the case of measurements of $q$, the angles correspond to $\alpha=0^{\circ}$ and $45^{\circ}$ and, for $u$, the settings are $\alpha=22.5$ and $67^{\circ} .5$, leading to

$q=\frac{\mathcal{I}(0)-\mathcal{I}(45)}{\mathcal{I}(0)+\mathcal{I}(45)} \quad$ and $\quad u=\frac{\mathcal{I}(22.5)-I(67.5)}{\mathcal{I}(22.5)+\mathcal{I}(67.5)}$

Consider the determination of $q$ from the signal given by Eq. (9) in its complete form.

$\alpha=0^{\circ}: \cos 2 \alpha=\cos 4 \alpha=1 ; \sin 2 \alpha=\sin 4 \alpha=0 \Longrightarrow$

$\mathcal{I}(0)=\frac{1}{2}\left\{k_{1}(I+Q)+k_{2}(I+Q)\right\}$.

$\underline{\alpha=45^{\circ}}: \cos 2 \alpha=\sin 4 \alpha=0 ; \sin 2 \alpha=1, \cos 4 \alpha=-1 \Longrightarrow$

$I(45)=\frac{1}{2}\left\{k_{1}(I-Q)+k_{2} U+\left(k_{1}+k_{3} \cos \Delta\right) Q\right\}$.

The normalised Stokes parameter, $q$, as evaluated by Eq. (11) is therefore given by

$q=\frac{k_{1} Q+k_{2}(I+Q-U)-k_{3} Q \cos \Delta}{2 k_{1} I+k_{2}(I+Q+U)+\left(k_{1}+k_{3} \cos \Delta\right) Q}$.

As a check, for a perfect wave plate, with $k_{1}=k_{3}=1, k_{2}=0$ and $\cos \Delta=-1$, the determined value simply reduces to $Q / I$.

If $Q, U$ are both zero, say by observing an unpolarized standard star, the apparent "instrumental polarization" associated with $q$ would be determined as

$\frac{k_{2}}{2 k_{1}+k_{2}}=\frac{\mathcal{T}_{\mathrm{f}}-\mathcal{T}_{\mathrm{s}}}{3 \mathcal{T}_{\mathrm{f}}+\mathcal{T}_{\mathrm{s}}}$

As this off-set depends on $k_{2}$, its origin is the polarizance of the wave plate. In general polarimetric reductions, it would be normal practice to simply subtract this generated instrumental off-set from other records from which unknown values of $q$ are being determined, but the form of Eq. (14) shows that this is invalid when high levels of accuracy are sought. In addition to the term $k_{2}(I+Q)$ being present both in the numerator and denominator, which in itself makes a straight forward subtraction incorrect, a term $k_{3} \cos \Delta$, related to the modulation efficiency, is present; its effects can be neglected only if $k_{1}=k_{3}$ and $\cos \Delta=-1$. For convenience, the spurious terms involving $Q$ in Eq. (14) may be referred to as resulting from "selfleakage". It may also be noted that the term $k_{2} U$ is present in the numerator and denominator of Eq. (14), the polarizance allowing parameter "cross-talk" to occur.

Similarly the value of $u$ would be determined as

$u=\frac{2 k_{1} U+\sqrt{2} k_{2}(I+Q)-\left(k_{1}+k_{3} \cos \Delta\right) U}{2 k_{1} I+\sqrt{2} k_{2} U+\left(k_{1}+k_{3} \cos \Delta\right) Q}$

Again, if $Q, U$ are both zero, the apparent "instrumental polarization" would be determined as

$\frac{k_{2}}{\sqrt{2} k_{1}}=\frac{\mathcal{T}_{\mathrm{f}}-\mathcal{T}_{\mathrm{s}}}{\sqrt{2}\left(\mathcal{T}_{\mathrm{f}}+\mathcal{T}_{\mathrm{s}}\right)}$.

When values of $u$ are determined, this instrumental polarization again cannot be simply subtracted to provide clean values. It can be seen from Eq. (16) that the polarizance introduces crosstalk from $Q$ in the numerator and self-leakage of $U$ in the denominator. The modulation efficiency involving, $\left(k_{3} \cos \Delta\right)$, introduces self-leakage into the numerator and cross-talk from $Q$ in the denominator.

By considering the test half-wave plate as described earlier, a section of the spectral behaviour of the instrumental polarization corresponding to the input light carrying zero polarization, i.e. $Q=U=0$, as determined from Eqs. (15) and (17) is depicted in Fig. 3. It can be seen that the apparent $q$ and $u$ parameters have similar behaviour but with differing amplitudes.

To investigate the strengths of effects associated with selfleakage and cross-talk, a range of $q$ and $u$ values for the input light was taken and the expected recorded values from Eqs. (14) and (16) determined and then "corrected" by subtracting the instrumental polarization as above. The difference between these results and the underlying true values was then determined in the form of a residual corresponding to a systematic error associated with a standard measurement and reduction procedure.

Two examples from the exercise are highlighted in Fig. 4 where it can be seen that the determined values exhibit fringes 

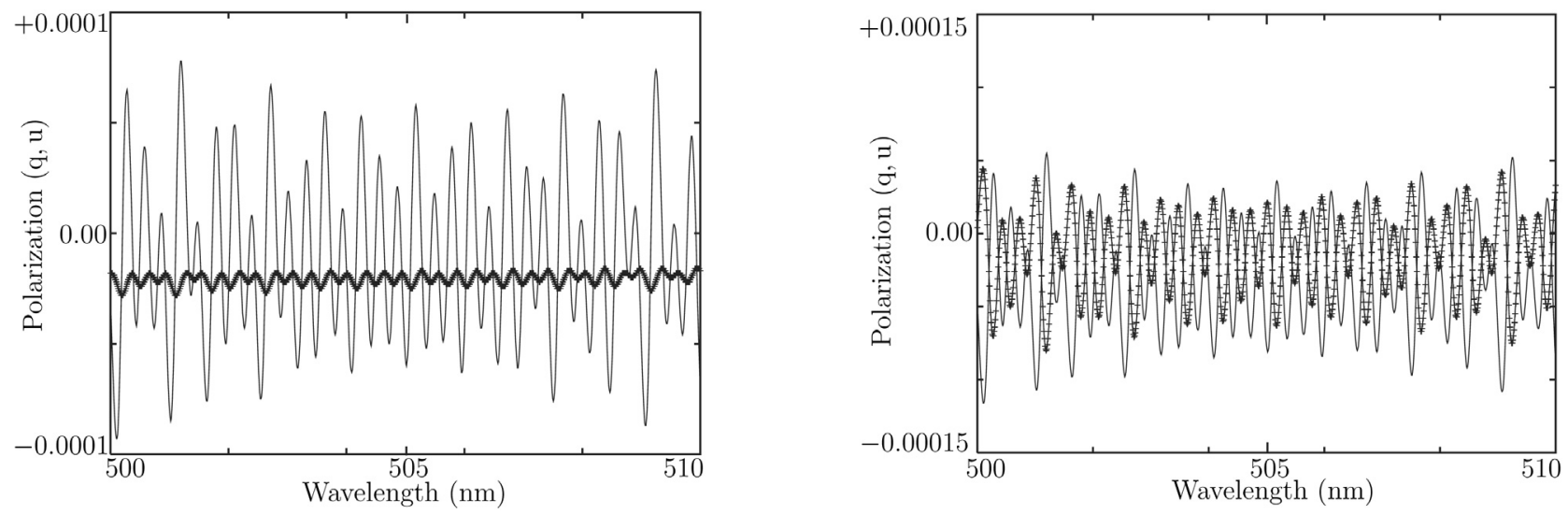

Fig. 4. The residual polarization for $q$ (heavy curves with marked points) and $u$ (light curves) for an achromatic half-wave plate over a spectral interval of $10 \mathrm{~nm}$. In the left hand figure the analysed light had wavelength independent values $q=0.01, u=0.0$; for the right hand figure, the input light had parameter values of $q=0.01, u=0.01$. Note that the mean level of these residuals is not zero, this resulting from the fact that the mean level of $\Delta$ is not $180^{\circ}$ over this wavelength section, being between the selected achromatic points.

which persist even after the instrumental polarization has been perfectly removed. When dealing with detections of linear polarization $\sim 0.01$, the fringe structures have amplitudes $\sim 0.0001$, suggesting that any polarization determinations are likely to be subject to a fringe noise of this order, so making it extremely difficult to record changes across spectral line features at polarimetric accuracies better than \pm 0.0001 .

In high accuracy spectropolarimetry, a full reduction procedure for the clean determination of $q$ and $u$ is only possible if the wavelength structure of the fringes of $k_{1}, k_{2}$ and $k_{3} \cos \Delta$ is known. With this information it should be possible to reduce the data by using iterative procedures commencing with trial values of $q$ and $u$. To determine all the instrumental parameters on the telescope, not only is it important to perform calibrations by observing stars presumed to offer unpolarized light, a selection of polarized standard stars covering a range of $p$ values and azimuths of vibration must be observed. It is also important to check out the stability of such measurements to see if temperature changes of the instrument on the telescope affect the fringe structure; Donati et al. (1999) have commented that the fringe patterns observed using one particular plate in their instrument varied significantly in position and shape on a timescale of only a few hours, probably as a result of temperature changes in the dome.

Obviously the severity of fringe problems depends on the detailed construction of the wave plate, whether anti-reflection coatings are used, whether the elements of an achromatic plate are cemented or in optical contact, etc. Over some parts of the overall spectral domain, the fringes associated with $\mathcal{T}_{\mathrm{f}}$ and $\mathcal{T}_{\mathrm{s}}$ may be in anti-phase producing a high polarizance with all the problems this introduces; in other spectral zones, the fringes associated with $\mathcal{T}_{\text {f }}$ and $\mathcal{T}_{\mathrm{s}}$ may be in phase, so minimising the problems caused by polarizance. It may well be that the differences in severity of the fringe effects associated with wave plates obtained from different manufacturers, as commented on by Donati et al. (1999), may be related to this.

From the above, it can be seen that the chief problem to measurements of linear polarization result from the polarizance of the wave plate as summarised in the third line of Eq. (9). It may be noted that these spurious signals have a modulation rate of $2 \alpha$ being half that of the designed signal contained in the first line of the same equation. As it turns out the signal recording procedure of Klare et al. (1972) operates as a heterodyne tuned to the modulation rate of $4 \alpha$ (see Clarke $\&$ Fullerton 1996); any spurious signals with other frequencies are automatically integrated out of the records (see Stewart 1985, for details for recording the data). It is perhaps for this reason that fringe effects were not detected in the spectropolarimetry undertaken at Glasgow University Observatory (e.g. see Clarke \& Brooks 1984, 1985; Clarke et al. 1985; Clarke \& Fullerton 1996). Consequently there are also strategies which eliminate the spurious $2 \alpha$ signals appropriate to spectropolarimetry using CCD detectors to record data at fixed positions of the half-wave plate.

Suppose that measurements are made at fixed positions of $\alpha$ of $0, \pi / 4, \pi / 2$ and $3 \pi / 4$. If $V$ is again assumed to be zero, the resulting records may be expressed as

$$
\begin{aligned}
& 2 \mathcal{I}(0)=k_{1}(I+Q)+k_{2}(I+Q) \\
& 2 \mathcal{I}\left(\frac{\pi}{4}\right)=k_{1}(I-Q)+k_{2} U+\left(k_{1}+k_{3} \cos \Delta\right) Q \\
& 2 \mathcal{I}\left(\frac{\pi}{2}\right)=k_{1}(I+Q)-k_{2}(I+Q) \\
& 2 \mathcal{I}\left(\frac{3 \pi}{4}\right)=k_{1}(I-Q)-k_{2} U+\left(k_{1}+k_{3} \cos \Delta\right) Q .
\end{aligned}
$$

Adding Eqs. (18) to (20) and Eqs. (19) to (21) leads to values of $2 k_{1}(I+Q)$ and $2\left(k_{1}(I-Q)+k_{3} Q \cos \Delta\right)$. Note that the polarizance related to $k_{2}$ does not appear in these identities. By adding these, and also subtracting one from the other, taking the ratio of the resultants leads to a determination of the first normalised Stokes parameter as

$q=\frac{\left(k_{1}-k_{3} \cos \Delta\right) Q}{2 k_{1} I+\left(k_{1}+k_{3} \cos \Delta\right) Q}$.

Similarly, by recording the signal at positions of the half-wave plate of $\alpha$ of $\pi / 8,3 \pi / 8,5 \pi / 8$ and $7 \pi / 8$, the determination of the second normalised Stokes parameter provides

$u=\frac{\left(k_{1}-k_{3} \cos \Delta\right) U}{2 k_{1} I+\left(k_{1}+k_{3} \cos \Delta\right) Q}$. 

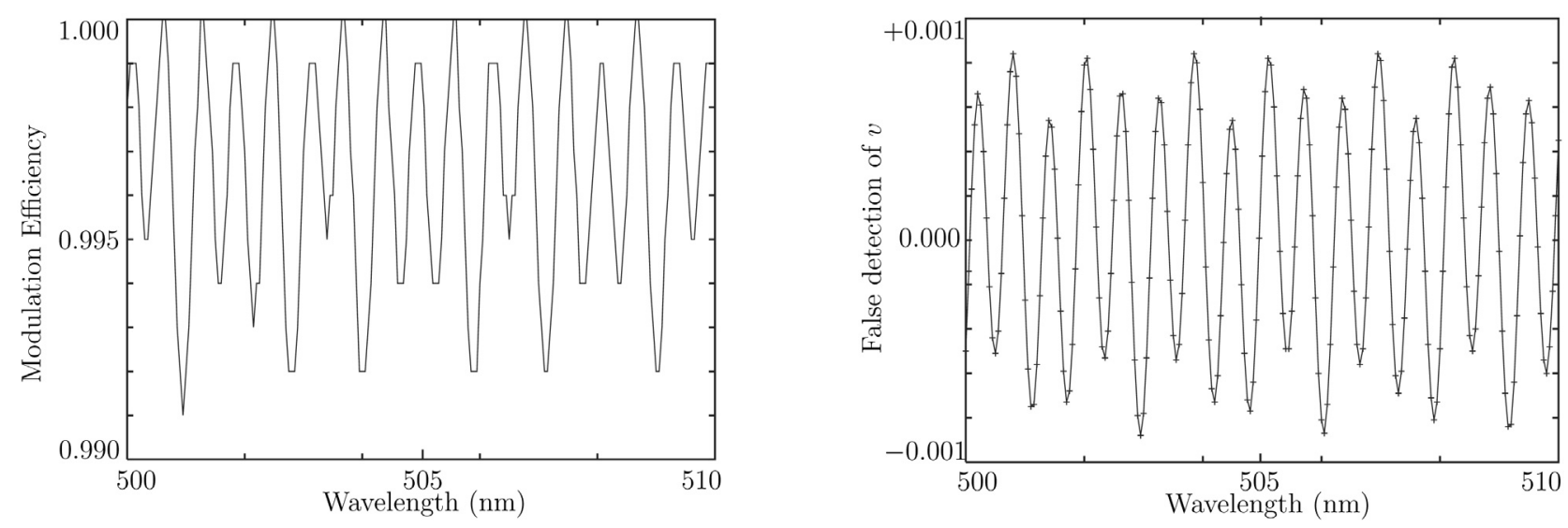

Fig. 5. The efficiency of detection of circularly polarized light is subject to complex spectral fringe structures as displayed in the left hand figure. In the right hand figure, fringes associated with the false detection of circularly polarized light is illustrated for a light source carrying a non-wavelength dependent linear polarization of $q=0.00, u=0.01$.

Relative to Eqs. (14) and (16), the new Eqs. (22) and (23) are much simpler and do not carry the effects of fringing associated with the wave plate's polarizance. It is interesting to note that if the system were to be calibrated by measuring unpolarized standard stars, the determined values of $q$ and $u$ should reduce to zero. Any measured non-zero values would correspond to other instrumental polarization such as from the telescope mirrors and should not exhibit fringing. The determined value of $q$ depends on $Q$ itself (self-leakage); similarly for $u$, there is a self-leakage term, but with additional crosstalk from $Q$. Calibration to allow the full corrections to be made to the reduced data again requires observations of standard stars with polarizations covering a range of values and azimuths of vibration.

\subsection{Measurements of circular polarization}

The standard wave plate for inclusion in a polarimeter designed to detect circular polarization has a retardation of $\pi / 2$. If again its polarizance is neglected such that $k_{1}=k_{3}=1, k_{2}=0$, and no linear polarization is present in the source, the signal given by Eq. (9) reduces to

$I(\alpha)=\frac{1}{2} I\{1-v \sin 2 \alpha\}$.

A standard technique for recording data is to make two measurements with $\alpha$ set at $\pm 45^{\circ}$.

$\underline{\alpha=-45^{\circ}} \quad \sin 2 \alpha=-1, \cos 2 \alpha=0$ :

$\mathcal{I}(-45)=I k_{1}-U k_{2}+Q k_{3} \cos \Delta+V k_{3} \sin \Delta$

$\underline{\alpha=+45^{\circ}} \quad \sin 2 \alpha=+1, \cos 2 \alpha=0$ :

$\mathcal{I}(+45)=I k_{1}+U k_{2}+Q k_{3} \cos \Delta-V k_{3} \sin \Delta$.

Subtracting Eqs. (26) from (25) gives

$-2 U k_{2}+2 V k_{3} \sin \Delta$.

Adding Eqs. (25) and (26) gives

$2 I k_{1}+2 Q k_{3} \cos \Delta$.
By dividing Eqs. (27) by (28), the determined degree of circular polarization would be given by

$v=\frac{V k_{3} \sin \Delta-U k_{2}}{I k_{1}+Q k_{3} \cos \Delta}$.

Again, for a perfect wave plate with $k_{1}=k_{3}=1, k_{2}=$ $0, \sin \Delta=1$ and $\cos \Delta=0$, the determined value of $v$ reduces to

$v=\frac{V}{I}$

If $Q, U$ are both zero, the determined value of $v$ is

$v=\frac{V k_{3} \sin \Delta}{I k_{1}}=\frac{2 V \sqrt{\mathcal{T}_{\mathrm{f}} \mathcal{T}_{\mathrm{s}}} \sin \Delta}{I\left(\mathcal{T}_{\mathrm{f}}+\mathcal{T}_{\mathrm{s}}\right)}$.

Thus, any spectrally determined values of $v$ are subject to fringes with form dictated by the transmittance of the wave plate and by $\Delta$. The value of $2 \sqrt{\mathcal{T}_{\mathrm{f}} \mathcal{T}_{\mathrm{s}}} \sin \Delta /\left(\mathcal{T}_{\mathrm{f}}+\mathcal{T}_{\mathrm{s}}\right)$ essentially represents the modulation efficiency to detect circularly polarized light and is subject to spectral fringes in response to the behaviour of $\mathcal{T}_{\mathrm{f}}, \mathcal{T}_{\mathrm{s}}$ and $\Delta$. Based on the design of the test achromatic quarter-wave plate described earlier, Fig. 5 displays the modulation efficiency over a small part of the spectrum, the fringe structure exhibiting a complicated pattern.

It is quite general that any target object is likely to carry linear polarization and the cross-talk from this is the biggest problem to measurements of circular polarization. With the experimental scheme outlined above it is the $u$ parameter which has the greatest affect on the measurements of circular polarization. The presence of any $q$ component has marginal effect on the determined values of $v$ as the cross-talk from it appears in the denominator rather than the numerator in the evaluation (see Eq. (29)). The right-hand panel of Fig. 5 displays the fringes related to the apparent spectral behaviour of $v$ for a typical monitored source carrying linear polarization with $u=0.01$.

\section{Conclusion}

In many spectropolarimeters, rotatable wave plates are used as part of the modulator system. Unfortunately when high accuracy data are pursued, the raw results tend to display spurious 
fringes. Their origin, as Harries \& Howarth (1996) have suggested, is likely to be caused by interference effects within the wave plate. Such effects, as shown by Clarke (2004a,b), give rise to fringes occurring in the plate's transmission, its polarizance and its phase retardation. In compound plates such as achromatic devices, the fringe structures are complex. Fringe structures also occur in the angle describing the axial frame of superachromatic devices of the Pancharatnam design (see Clarke 2004c), this in turn giving rise to fringes in the angle describing the co-ordinate frame in which the measured Stokes parameters are described.

The strengths of all the various fringes and their effect on polarimetric measurements depends on the design and manufacture of the wave plate, important considerations include the choice of anti-reflection coatings and whether the elements of compound plates are bonded or just simply in optical contact.

By investigating their action in the detection of both linear and circular polarization, it is demonstrated that the major problem is the fringe structures associated with the plate's polarizance. In the case of achromatic devices, it should be possible to design compound plates with elements of the correct thickness to achieve achromatism but at the same time controlling the fringes associated with the orthogonal transmissions to be in phase with each other over the major part of the available band-pass. Polarizance fringes can be reduced by antireflection coatings; their effects might also be reduced by locating the wave plate in a medium with refractive index close to the indices of the plate's material rather than in air. It has also be shown that, in the case of linear polarization measurements, the deleterious effects of polarizance fringes can be removed by particular selection of position angles of the wave plate in the experimental procedure.

For a complete data reduction involving the removal of effects of cross-talk between the various Stokes parameters, the usual measurements of standard stars (unpolarized and polarized) need to be undertaken but it is important to monitor several polarized standards with position angles differing by $45^{\circ}$, so that values of $k_{1}, k_{2}$ and $k_{3} \cos \Delta$ may be well determined; their effects on the $q$ and $u$ values of target stars may be removed by iterative procedures. Information on the effects of temperature on the birefringence of materials and on the behaviour of basic properties of wave plates is sparse. In view of comments made by Donati et al. (1999), the newly encountered fringe structures and their stability with respect to temperature require exploration. It would seem prudent, however, to house any modulator in a temperature controlled cell. This has always been standard practice in instruments developed at Glasgow University Observatory.

\section{References}

Clarke, D. 1967, Optica Acta, 14, 343

Clarke, D. 2004a, J. Opt. A: Pure Appl. Opt., 6, 1036

Clarke, D. 2004b, J. Opt. A: Pure Appl. Opt., 6, 1041

Clarke, D. 2004c, J. Opt. A: Pure Appl. Opt., 6, 1047

Clarke, D., \& Grainger, J. F. 1971, Polarized Light and Optical Measurement (Oxford: Pergamon Press)

Clarke, D., \& Brooks, A. 1984, MNRAS, 211, 737

Clarke, D., \& Brooks, A. 1985, MNRAS, 212, 211

Clarke, D., Schwarz, H. E., \& Stewart, B. G. 1985, A\&A, 145, 232

Clarke, D., \& Fullerton, S. R. 1996, A\&A, 310, 331

Clarke, D., \& Neumayer, D. 2002, A\&A, 383, 360

Donati, J.-F., Catala, C., Wade, G. A., et al. 1999, A\&AS, 134, 149

Fessenkov, V. G. 1935, AZh, XII, (4), 309

Harries, T. J., \& Howarth, I. D. 1996, A\&A, 310, 533

Klare, G., Neckel, Th., \& Schnur, G. 1972, A\&AS, 5, 539

Pancharatnam, S. 1955, Proc. Indian Acad. Sci. A, 41, 137

Pickering, E. C. 1874. Proc. Am. Acad. of Arts and Sciences, IX, 1

Semel, M. 2003, A\&A, 401, 1

Serkowski, K. 1974, Polarization Techniques - Chapter 8 from Methods of Experimental Physics, ed. M. L. Meeks, \& N. P. Carleton, 12, Astrophysics: Part A, Optical \& Infrared (Academic Press)

Stewart, B. G. 1985, A\&A, 143, 235

Tinbergen, J. 1994, Spectrum - Newsletter of the Royal Observatories, 1,26

Tropf, W. J., Thomas, M. E., \& Harris, T. J. 1995, Properties of Crystals and Glasses, Chapt. 33 in Handbook of Optics 2 (New York: McGraw Hill) 